\title{
Simulation and experimental research on micro-channel for detecting cell status in bio-artificial liver
}

\author{
Changzhe $\mathrm{Wu}$, Yue Cao, Xiaolin Huo* and Ming Li \\ Beijing Key Laboratory of Bioelectromagnetism, Institute of Electrical Engineering, Chinese Academy \\ of Sciences, Beijing, China
}

\begin{abstract}
.
BACKGROUND: Bioartificial liver support system (BALSS) based on culturing hepatocytes is an important research field for the treatment of acute liver failure. It is necessary to monitor the state of liver cell functions during the treatment of BALSS in order to guide clinical treatment.

OBJECTIVE: To design a micro-channel chip to achieve flash mixing for timely detection of liver cell status in bioreactors and improving liver cells growth environment to ensure the efficacy of the bio-artificial liver support system.

METHODS: Alanine aminotransferase (ALT) and Urea are chosen as detection indicators to reflect the degree of liver cell injury and the detoxification function. A diamond tandem structure micro-channel is designed and optimized to achieve the efficient mixing of serum and ALT or Urea reagent.

RESULTS: The simulation and experimental results show that the diamond tandem structure micro-channel can significantly improve the mixing efficiency and meet the online detecting requirements.

CONCLUSION: The easily controllable diamond tandem structure micro-channel combines the advantages of active and passive mixer and can effectively mix the serum and ALT or Urea reagent. It lays the foundation for online monitoring of liver cells and will help to improve the viability of liver cell in the bioreactor.
\end{abstract}

Keywords: Bio-artificial liver, ALT, urea, ANSYS, mixing efficiency

\section{Introduction}

Liver failure is a disease with a mortality rate of up to $80 \%$. Bio-artificial livers are used in the treatment of patients who suffer from liver failures. The core of a bio-artificial liver is the bioreactor, where liver cells grow and function. Therefore, the microenvironment in bioreactor is directly related to the efficacy of the bio-artificial liver. Currently, the detection of the status of liver cells in bioreactors is conducted offline and the operations are cumbersome, and the feedback of which cannot be directly used to adjust the microenvironment in a timely manner. In this paper, we designed an innovative micro-channel, which can help evaluate the liver cell status in real time. Based on the principle of optical colorimetry, Alanine aminotransferase (ALT) and Urea were chosen as detection indicators to reflect the degree of

\footnotetext{
${ }^{*}$ Corresponding author: Xiaolin Huo, Beijing Key Laboratory of Bioelectromagnetism, Institute of Electrical Engineering, Chinese Academy of Sciences, Beijing, China. Tel.: +86 010 82547242; Fax: +86 010 82547242; E-mail: huoxl@mail.iee.ac.cn.
} 
liver cell injury and the detoxification function. A spectrometer was used to detect the change of absorbance at wavelength of $340 \mathrm{~nm}$ caused by the concentration of ALT or Urea [1]. The concentration of ALT and Urea could accurately reflect liver cell growth and functioning status, and therefore are important indicators for bio-artificial livers to improve efficacy.

Since serum transfusion often exposes the liver cells to infection, in long-term cell status monitoring, it is necessary to minimize the usage of micro-channel which consumes serum in the detections This micro-channel needs to achieve efficient mixed reaction to ensure the reliability of online detecting. Common micro-mixing methods can be classified into two categories: active mixing and passive mixing. Active mixing requires external energy, such as magnetic, acoustic field force or electric force [2]. Bruyker et al. [3] designed an active mixing based on magnetic actuation using micro machined magnetic stir bars last year. Ryu et al. [4] designed a micro magnetic stir-bar integrated in parylene. Although both of them have high efficiency and small mixed-scale, they need complex processing and integration, which made them difficult to be widely applicable. Due to its simplicity and easy fabrication, passive mixing is an more attractive solution for most cases. Common types of passive mixers include: T/Y type micro-mixer [5]6], wavy channel micro-mixer, micro-mixer with static resistance, and so on. Sui [7] simulated a wavy channel micro-mixer to enhance mixing by increasing Reynolds numbers. Naher [8] and $\mathrm{Xu}$ [9] optimized mixing effect by comparing different kinds of obstacle structures. Jeon [10] designed a zigzag type micro-mixer, and proved that its mixing effect is better than the micro-mixer with static obstacle. These structures still cannot meet our requirements of mixing. Although their structures are different, their principles are the same to improve mixing effect by changing geometric structure to increase turbulence or to reduce the diffusion distance.

In the present study, we designed a diamond tandem structure micro-channel which can repeat the cycle of split-and-recombine to achieve better mixing. Simulation software ANSYS14.0-Fluent was used to optimize the structure [11]. Experiments were carried out to verify the effectiveness of the microchannel.

\section{Theory and modeling}

The structure of diamond tandem micro-channel is shown in Fig. 1. The length of the model is $4 \mathrm{~cm}$. Section 1 represents the reagent inlet and Section 2 represents the serum inlet. In order to facilitate the matching of the inlet pipe diameter, the inlets of the micro channel width is set to $800 \mu \mathrm{m}$. The width of the subsequent inlets were decreased progressively to $500 \mu \mathrm{m}, 300 \mu \mathrm{m}, 200 \mu \mathrm{m}, 150 \mu \mathrm{m}$ and finally to the standard width of $100 \mu \mathrm{m}$. The ratio of the width of the micro-channel to the depth is generally less than 5:1 in order to avoid the deformation and depression. Based on this, the micro-channel depth was set to $200 \mu \mathrm{m}$. We used hexahedral grid to mesh the micro-channel and set the boundary layer grid. The maximum size of grids was $10 \mu \mathrm{m}$ and the total number of grids was more than 3 million.

The designing idea is shown in Fig. 2. Serum is represented by the color red and ALT or Urea detection reagent is represented by the color blue. The liquid from the straight-shaped channel flows into the rhombus-shaped channel and was divided into two branches. The angle between the two branches is $90^{\circ}$. As shown in Fig. 2, the flow velocity $V$ can be decomposed into $V_{x}$ and $V_{y}$. The direction of the flow velocity $V_{y}$ is consistent with the direction of two liquids' concentration gradient, which can speed diffusion efficiency of two liquids in the direction of $y$ [12]. The liquid flows into straight-shaped channel after outflows from the rhombus-shaped channel, the flow velocity $V$ can also be decomposed into $V_{x}$ and $V_{y}$. The direction of the flow velocity $V_{y}$ is consistent with the direction of two liquids' concentration gradient, which can speed diffusion efficiency of two liquids in the direction of $y$ too. 


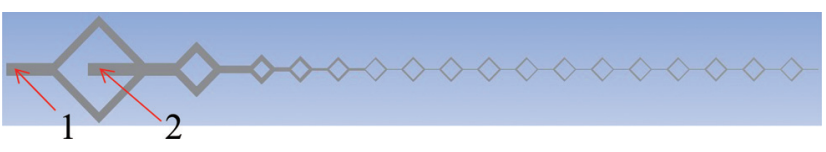

Fig. 1. Schematic diagram of the diamond tandem micro-channel.

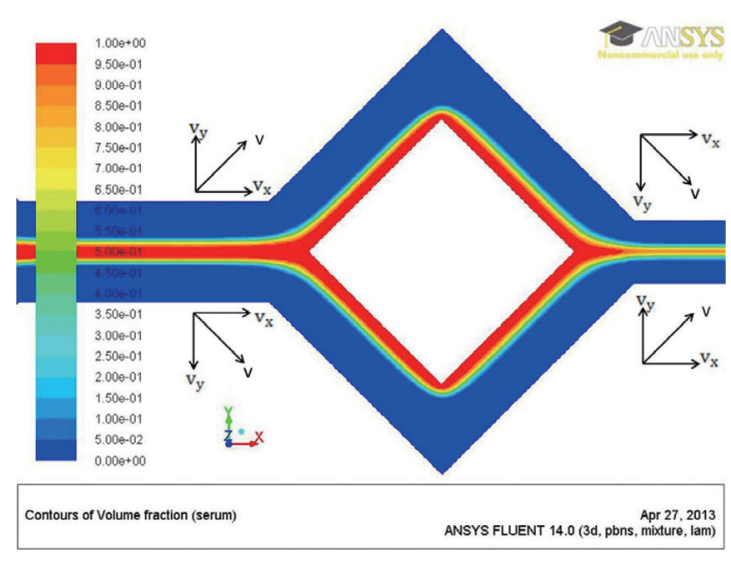

Fig. 2. Schematic of design idea.

More model elements in series can make the liquid constantly separate and merge, thus creating more opportunities for the concentration gradient and flow velocity $V_{y}$ in the same direction to improve the mixing efficiency. Based on this principle, we can largely improve the mixing efficiency by increasing the number of the model element.

The length of the simulation model is $4 \mathrm{~cm}$, as shown in Fig. 1. The commercial software ANSYS14.0Fluent is employed in the numerical simulation. Computer simulation to Inter CORE i5 dual-core processor, $16 \mathrm{G}$ memory, computation time is different for each model, about 3 days -4 days. The three dimensional Navier-Stokes equations were solved. The liquids need to be mixed in micro-channel are detection reagent and serum. Serum density is $\rho_{\text {serum }}=1042.25 \mathrm{~kg} / \mathrm{m}^{3}$ and viscosity is $\mu_{\text {serum }}=4.15$ $\times 10^{-3} \mathrm{~kg} / \mathrm{m} \cdot \mathrm{s}$. The density, viscosity and other characteristics of ALT and Urea detection reagent are close to those of water. We use water to replace two kinds of reagent in the simulation. Reagent density is $\rho_{\text {reagent }}=998.2 \mathrm{~kg} / \mathrm{m}^{3}$ and viscosity is $\mu_{\text {reagent }}=1.003 \times 10^{-3} \mathrm{~kg} / \mathrm{m} \cdot \mathrm{s}$. Serum and detection reagent are assumed to be newtonian fluid. The micro-channel characteristic scale is the micron level and the reynolds number is far less than 2000. Laminar model and multiphase mixture model are used [13]. The velocity-inlet and pressure-outlet boundaries are used. The flow rate of Vserum is $2.5 \mu \mathrm{l} / \mathrm{min}$, VALT reagent is $25 \mu \mathrm{l} / \mathrm{min}$ and VUrea reagent is $250 \mu \mathrm{l} / \mathrm{min}$.

Mixing efficiency $\alpha[14]$ and minimum mixing ratio $\eta$ were chosen to reflect the degree of mixing. Mixing efficiency $\alpha$ is defined as:

$$
\alpha=\left(1-\frac{\sigma}{x}\right) \times 100 \%
$$

Where $x$ is the ideal value of the mixing serum. Due to the flow ratio of $10: 1$ between ALT reagent and serum volume, the ideal value $x$ is $9.1 \%$ when detecting ALT. When detecting Urea, the serum ideal mixing value $x$ is $0.99 \%$, due to Urea reagent and serum volume flow ratio is $100: 1$. Where $\sigma$ is standard deviation.

$$
\sigma=\sqrt{\frac{\sum_{i=1}^{n}\left(c_{i}-x\right)^{2}}{n}}
$$

Where $c_{i}$ is serum volume distribution value in every compute node and $n$ is the total number of all nodes in the computational domain. 

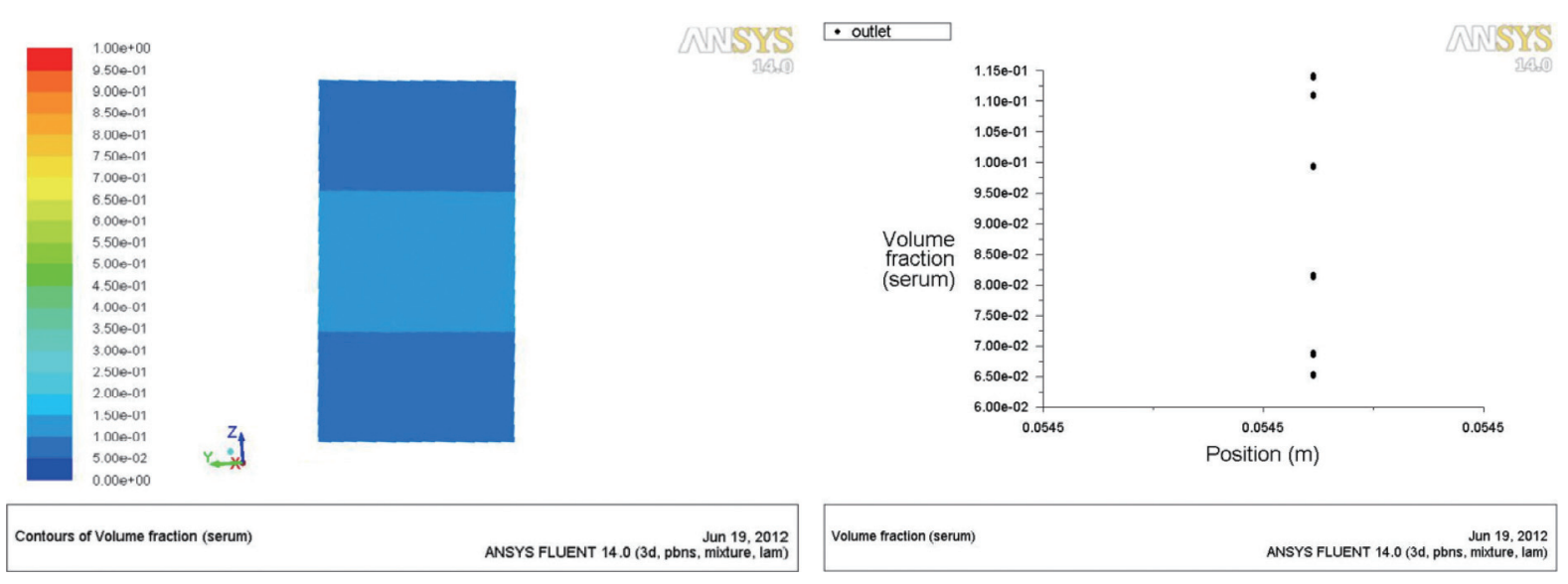

Fig. 3. Serum volume fraction contours of outlet.

Minimum mixing ratio $\eta$ is defined as:

$$
\eta=\min \left\{\left(1-\frac{\sqrt{\left(x-c_{i}\right)^{2}}}{x}\right) \times 100 \%\right\}, \quad i \in[1, n]
$$

\section{Simulation and analysis}

The mixing effect of ALT reagent and serum using the simulation model were as shown in Fig. 1, the flow rate $V$ of the serum (Vserum) was $2.5 \mu \mathrm{l} / \mathrm{min}$ and the flow rate of ALT (VALT) reagent was $25 \mu \mathrm{l} / \mathrm{min}$. The velocity-inlet and pressure-outlet boundaries were used. The wall was adiabatic with zero heat flux and the boundary was no-slip. Steady solver was used. After entire computational domain was initialized to be filled with serum, simulation was started. As shown in Fig. 3, the serum volume fraction of outlet range was from $6 \%$ to $11.5 \%$. Mixing efficiency $\alpha$ is $78.9 \%$ and minimum mixing ratio $\eta$ was $66 \%$, which did not achieve complete mixture. The model needed to be improved.

As shown in Fig. 3, the serum was mainly located in the upper and lower hierarchical directions, and was unevenly distributed in the outlet. Therefore, we changed the model vertical structure to optimize the mixing effect. Comparisons of established models are shown in Fig. 4.

Comparing the four models in Fig. 4, we can discover the main difference with the original diamond tandem structure channel lies in vertical structure. In model A, the height of the straight-shaped channel was reduced to $100 \mu \mathrm{m}$. In model B, the height of the rhombus-shaped channel was reduced to $100 \mu \mathrm{m}$. Model $\mathrm{C}$ reduced all the height of the original diamond tandem structure channels to $100 \mu \mathrm{m}$. Model $\mathrm{D}$ reduced the height of the original diamond tandem structure channel to $100 \mu \mathrm{m}$ and progressively reduced the width to $500 \mu \mathrm{m}, 300 \mu \mathrm{m}, 200 \mu \mathrm{m}, 150 \mu \mathrm{m}$ and $100 \mu \mathrm{m}$, respectively. According to the results of the simulation, the mixing effect of model $\mathrm{C}$ is best. The outlet serum volume fraction of model $\mathrm{C}$ ranged from $8.7 \%$ to $9.3 \%$. Mixing efficiency $\alpha$ was $97.69 \%$ and the minimum mixing ratio $\eta$ was $95.6 \%$, which met the requirements of ALT online detecting. Model A, B and D failed to meet the threshold of the $95 \%$ mixing efficiency $\alpha$ and the $95 \%$ minimum mixing ratio $\eta$. The micro-channel chip was able to process in real time. Therefore, model $\mathrm{C}$ was chosen as the optimized micro-channel. 


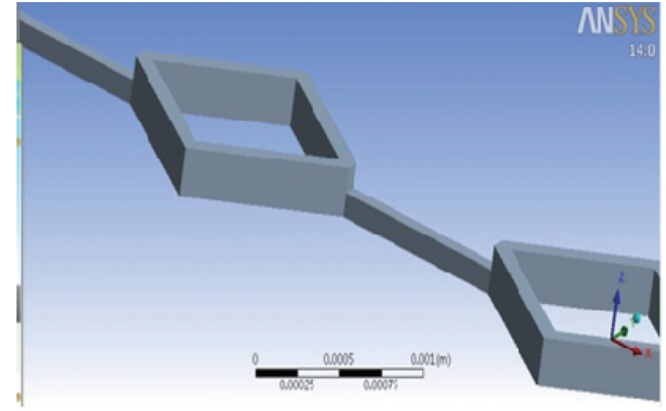

(A)

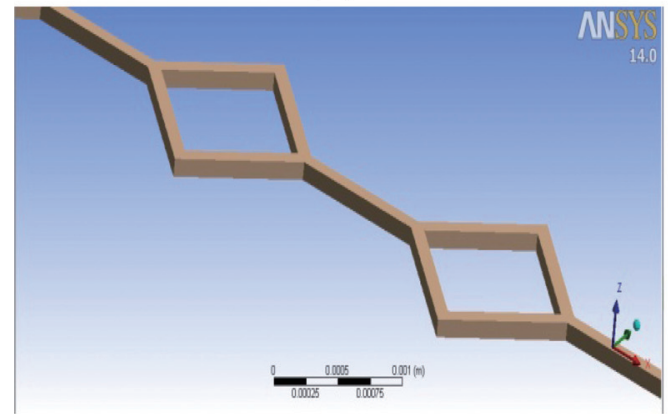

(C)

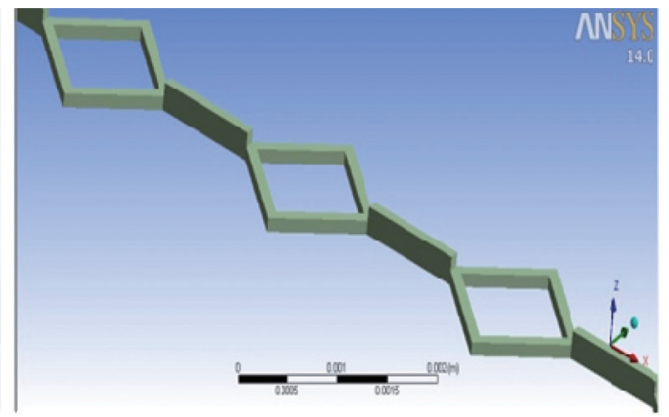

(B)

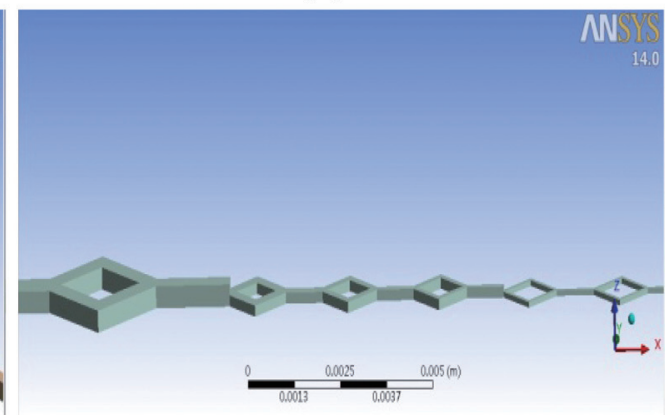

(D)

Fig. 4. Optimal comparison models.

The mixing effect of Urea reagent and serum was investigated using the model $\mathrm{C}$, the flow rate Vserum was $2.5 \mu \mathrm{l} / \mathrm{min}$ and VUrea reagent was $250 \mu \mathrm{l} / \mathrm{min}$. As the flow rate increased about 10 times, the mixing effect was not satisfactory. So we extended length of model $\mathrm{C}$ to $12 \mathrm{~cm}$ to improve mixing effect. The outlet serum volume fraction ranged from $0.997 \%$ to $1.00 \%$. Mixing efficiency $\alpha$ was $99.97 \%$ and minimum mixing ratio $\eta$ was $98.9 \%$, which meet requirements of Urea online detecting.

\section{Experiment and discussion}

Based on the results of the simulation, we designed the diamond tandem structure micro-channel chip and detection platform for the detection of cell status in bio-artificial livers. The design is shown in Fig. 5 . The diamond tandem structure micro-channel chip was made of polydimethylsiloxane (PDMS) [4]. Diluted distributor, deuterium light source and the spectrometer were used for online detection with the diamond tandem structure micro-channel chip together.

To verify the detection accuracy of the micro-channel chip, 6 serum samples containing different concentrations of ALT or Urea were used.

The detection platform in Fig. 5, detected six different concentrations of ALT and Urea. The experimental results met the detection accuracy requirement of ALT and Urea. Linear fitting diagram of the experimental results are shown in Fig. 6.

Experimental results show that the detected values of the diamond tandem structure micro-channel chip were consistent with the reagents reference values. ALT linear correlation coefficient was 0.999787 and norm of residuals was $1.7541 \mathrm{U} / \mathrm{L}$. Urea linear correlation coefficient was 0.999829 and norm of 
Table 1

Serum samples

\begin{tabular}{lll}
\hline $\begin{array}{l}\text { Serum } \\
\text { samples }\end{array}$ & $\begin{array}{l}\text { Concentration } \\
\text { value of } \\
\text { ALT (U/L) }\end{array}$ & $\begin{array}{l}\text { Concentration } \\
\text { value of } \\
\text { urea (mmol/L) }\end{array}$ \\
\hline Level 1 & 17.65 & 2.55 \\
Level 2 & 35.3 & 5.1 \\
Level 3 & 53.9 & 6.68 \\
Level 4 & 72.5 & 8.26 \\
Level 5 & 96.25 & 9.165 \\
Level 6 & 120 & 10.07 \\
\hline
\end{tabular}
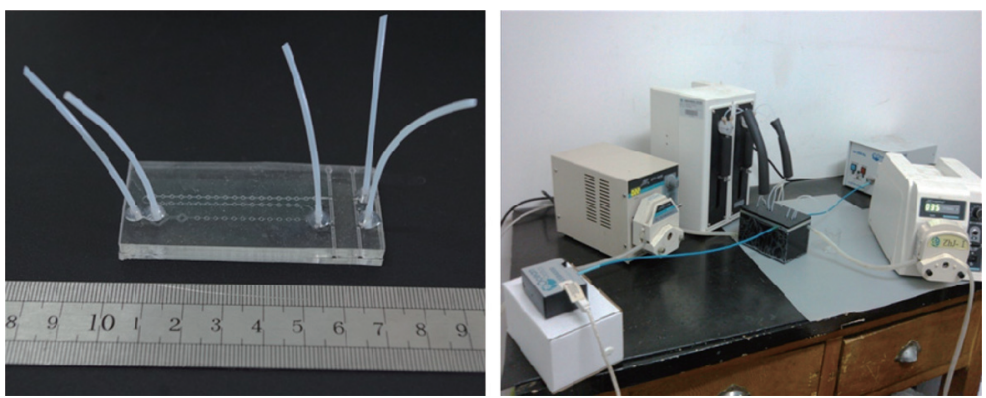

Fig. 5. The designed and processed micro-channel chip and detection platform.

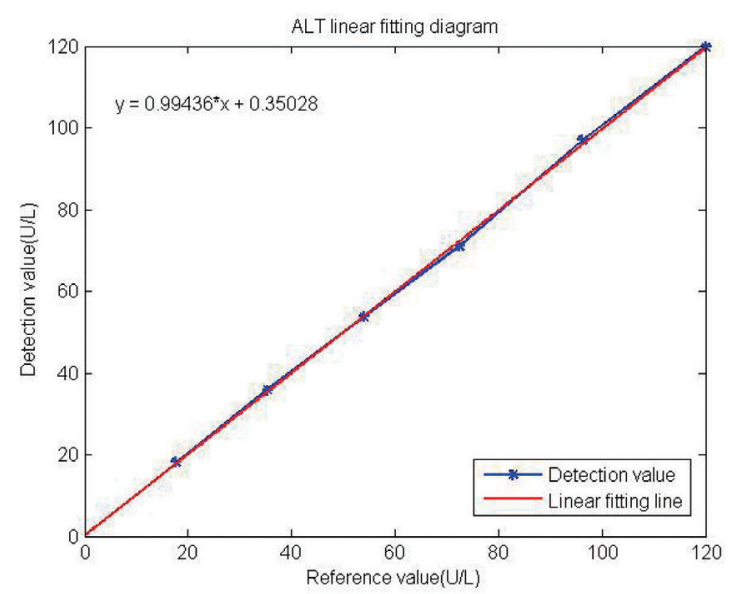

(A)

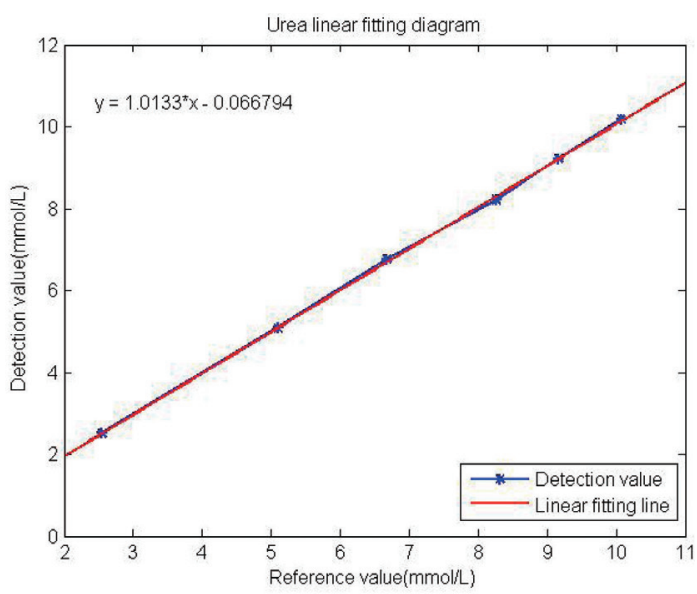

(B)

Fig. 6. Linear fitting diagram of the experimental results: (A) ALT (B) Urea.

residuals was $0.11728 \mathrm{mmol} / \mathrm{L}$. All of the detected values had good stability, which could meet detecting requirements. The results also had a good linear correlation. The micro-mixer chip could deliver flash mixing and detection for bio-artificial livers.

\section{Summary}

The diamond tandem structure micro-channel was designed to accomplish better mixing effect for detecting cell status in bio-artificial liver. The simulation indicated our design yielded satisfactory mixing effects. The micro-channel chip can largely improve mixing effect without external force, which can easily be used in many biological and chemical fields compared to active mixers. The micro-channel chip is made of polydimethylsiloxane (PDMS). The total volume of the micro-channel chip was about $17 \mu \mathrm{L}$. When detecting alanine aminotransferase (ALT), the serum consumption was only $1.55 \mu \mathrm{L}$. When detecting Urea, the serum consumption is only $0.1717 \mu \mathrm{L}$. Due to the little serum consumption, this micro-channel chip can be used in real time online monitoring of the cell status without the need of frequent transfusion to the patients. Using the online micro-detection method for liver cell in bioreactor, we can provide a long time, continuous, low consumption and automatic detection for patients. This 
method lays the foundation for optimizing the status of liver cell in bioreactor and is very important to improve the efficacy of the bio-artificial liver.

\section{Acknowledgments}

Research was financially supported by the National 863 Plan Project of China (Grant No. 2012AA 020505) and the National Natural Science Foundation of China (Grant No. 61101050).

\section{References}

[1] Y.W. Ye, Y.S. Wang and Z.Y. Shen: National guide to clinical laboratory procedures (Medical Administration Department of Ministry of Public Health, China 2006).

[2] B.C. Lin and J.H. Qin: Microfluidic lab on a chip (Science Publishing House, China 2008).

[3] D. De Bruyker, M.I. Recht, A.A.S. Bhagat, F.E. Torres, A.G. Bell and R.H. Bruce: Lab on a Chip, Vol. 11(2011), p. 3313-3319.

[4] K.S. Ryu, K. Shaikh, E. Goluch, Z. Fan and C. Liu: Lab Chip, Vol. 4(2004), p. 608-613.

[5] E.A. Mansur, M. Ye, Y. Wang and Y. Dai: Chinese Journal of Chemical Engineering, Vol. 16(2008), p. 14.

[6] S.M. Tu, S. Torii and Y.C. Shih: The International Journal of Multiphysics, Vol. 5(2011), p.215-228.

[7] Y. Sui, C. Teo and P. Lee: International Journal of Heat and Mass Transfer, Vol. 55(2011), p. 73-88.

[8] S. Naher, D. Orpen, D. Brabazon, C.R. Poulsen and M.M. Morshed: Simulation Modelling Practice and Theory, Vol. 19(2011), p. 1088-1095.

[9] Z. Xu, C. Li, D. Vadillo, X. Ruan and X. Fu: Sensors and Actuators B: Chemical, Vol. 153(2010), p. 284-292.

[10] L. Zhu, Y.L. Hou and W.Y. Zhang: MEMS Device and Technology, Vol. 42(2005), p. 164-171.

[11] Y. Cao, C.Z. Wu, X.L. Huo and M. Li: Applied Mechanics and Materials, Vol. 246(2013), p. 241-245.

[12] X.F. Zhu and Y.X. Lu: Journal of Engineering Thermophysics, Vol. 17(1996), p. 428-432.

[13] Y. Cao, M.M. Duan, C.Z. Wu, X.L. Huo and M. Li: Beijing Biomedical Engineering, Vol. 31(2012), p. 331-337.

[14] D. Lee and P.-H. Lo: Chemical Engineering Journal, Vol. 181-182(2012), p. 524-529. 